\title{
Energy and Symmetry-preserving formulation of nonlinear constraints and potential forces in multibody dynamics
}

\author{
$\underline{\text { Juan C. García Orden }}^{1}$ \\ ${ }^{1}$ Dep. de Mecánica de Medios Continuos y Teoría de Estructuras \\ ETSI Caminos, Canales y Puertos \\ Universidad Politécnica de Madrid \\ c/ Profesor Aranguren 3, 28040 Madrid (Spain) \\ juancarlos.garcia@upm.es
}

\section{EXTENDED ABSTRACT}

\section{Introduction}

Many mechanical problems are adequately represented by models composed by different elements such as particles, rigid and deformable bodies. A common feature of many of them is the presence of joints that limit or couple the motion of the different parts of the system. A proper representation of these joints in the model is essential in order to obtain an accurate solution, usually through specialized numerical schemes.

One category of such schemes is the so-called geometric integrators, designed to provide numerical solutions that exactly inherit basic features of the underlying time-continuous model. Some of these schemes, in the context of the dynamics of Hamiltonian systems, are the Energy-Momentum (EM) methods, that are second-order schemes that preserve the total energy in conservative mechanical problems and possible symmetries associated with the conservation of linear and angular momenta.

The concept of discrete derivative $[1,2]$ plays a central role in the systematic design of a EM method. Interestingly, its particular expression is not unique and accordingly several formulations have been proposed in the literature for our systems of interest. Nevertheless, not all of them reveal suitable for the EM formulation of completely general constraints and potential forces. Surprisingly, this fact seems to have been overlooked by many authors, and constitutes the main motivation of the developments presented here. The main result is that just one of the reviewed discrete derivatives generally complies with the requirements of a EM method, while the others do so only in particular cases.

\section{Time-continuous model: energy conservation and symmetries}

Let us consider a system $\mathscr{B}$ composed by $N$ particles with masses $m_{i}, i=1, \ldots, N$, moving in the three-dimensional Euclidean space. The motion of this system at time $t \in[0, T]$ is described by the vectors $\mathbf{q}(t), \mathbf{p}(t) \in \mathbb{R}^{3 N}$ that collects the cartesian inertial coordinates of their position and momentum vectors $\mathbf{r}_{i}(t), \mathbf{p}_{i}(t)=m_{i} \dot{\mathbf{r}}_{i} ; i=1, \ldots, N$. What is more, lets us assume that there are no external forces and that the motion of the system is constrained by a general holonomic constraint function $\Phi: \mathbb{R}^{3 N} \times[0, T] \rightarrow \mathbb{R}$, such that $\Phi(\mathbf{q}, t)=0$. The equations of motion are given by the index-3 DAE system:

$$
\begin{aligned}
\dot{\mathbf{q}} & =\mathbf{M}^{-1} \mathbf{p} \\
\dot{\mathbf{p}} & =-D_{\mathbf{q}} \Phi^{\mathrm{T}} \lambda \\
0 & =\Phi(\mathbf{q}, t)
\end{aligned}
$$

M being the mass matrix (diagonal), vector $D_{\mathbf{q}} \Phi=\partial \Phi / \partial \mathbf{q}$ and $\lambda \in \mathbb{R}$ a Lagrange multiplier.

It is easy to prove that the energy of the system is constant provided the constraint does not depend explicitly on time (i.e., it is a scleronomous constraint). Using (2) and taking into account that in this case $\dot{\Phi}=D_{\mathbf{q}} \Phi \dot{\mathbf{q}}=D_{\mathbf{q}} \Phi \mathbf{M}^{-1} \mathbf{p}=0$ :

$$
\begin{aligned}
& E=\frac{1}{2} \mathbf{p}^{\mathrm{T}} \mathbf{M}^{-1} \mathbf{p} \\
& \dot{E}=\mathbf{p}^{\mathrm{T}} \mathbf{M}^{-1} \dot{\mathbf{p}}=-\lambda \mathbf{p}^{\mathrm{T}} \mathbf{M}^{-1} D_{\mathbf{q}} \Phi^{\mathrm{T}}=0
\end{aligned}
$$

On the other hand, Noether's theorem states that a mechanical system has a conservation law for each symmetry of its evolution equations [6]. In our system, these symmetries may be translational and rotational, and the corresponding conserved magnitudes would be the linear and angular momenta in $O$, defined respectively as:

$$
\mathbf{L}=\sum_{i=1}^{N} \mathbf{p}_{i}=\mathbb{1} \mathbf{p} \quad, \quad \mathbf{J}=\sum_{i=1}^{N} \mathbf{r}_{i} \times \mathbf{p}_{i}=r \mathbf{p}
$$

$\mathbb{1}$ and $₫$ being the following $3 \times 3 N$ matrices:

$$
\mathbb{1}=(\mathbf{1}|\mathbf{1}| \ldots \mid \mathbf{1}) \quad, \quad \widetilde{r}=\left(\hat{\mathbf{r}}_{1}\left|\hat{\mathbf{r}}_{2}\right| \ldots \mid \hat{\mathbf{r}}_{N}\right)
$$


1 being the identity $3 \times 3$ matrix and $\hat{\mathbf{r}}_{i}$ denoting the $3 \times 3$ skew-symmetric matrix associated to vector $\mathbf{r}_{i}$. Differentiating (6), taking into account (2) and the fact that $\dot{\sim} \mathbf{p}=\mathbf{0}$, we obtain:

$$
\dot{\mathbf{L}}=\mathbb{1} \dot{\mathbf{p}}=-\lambda \mathbb{1} D_{\mathbf{q}} \Phi^{\mathrm{T}} \quad, \quad \dot{\mathbf{J}}=\llbracket \dot{\mathbf{p}}=-\lambda 匹 D_{\mathbf{q}} \Phi^{\mathrm{T}}
$$

Equations (8) reveal the orthogonality conditions to be met by a constraint to conserve the linear and angular momenta:

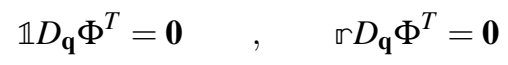

\section{EM formulation. Discrete energy conservation and symmetries}

We consider next the continuous problem given by (1)-(3) for the particular case of an holonomic and scleronomous constraint $\Phi(\mathbf{q})=0$, which is energy-conserving as justified in the previous section. Accordingly, in what follows we will drop the subscript $\mathbf{q}$ in the derivative $D_{\mathbf{q}} \Phi$ in order to simplify the notation, becoming $D \Phi$. The proposed EM formulation is described by the following second-order (in positions) time-stepping scheme, which at first glance can be interpreted as a modified implicit midpoint rule:

$$
\begin{aligned}
& \mathbf{q}_{n+1}-\mathbf{q}_{n}=\frac{\Delta t}{2} \mathbf{M}^{-1}\left(\mathbf{p}_{n}+\mathbf{p}_{n+1}\right) \\
& \mathbf{p}_{n+1}-\mathbf{p}_{n}=-\Delta t \lambda_{n+1} \mathrm{D} \Phi^{\mathrm{T}} \\
& \Phi_{n+1}=0
\end{aligned}
$$

$\mathbf{q}_{n}$ and $\mathbf{p}_{n}$ being the approximated values of $\mathbf{q}$ and $\mathbf{p}$ at $t_{n} \in[0, T]$ respectively, $\Phi_{n}=\Phi\left(\mathbf{q}_{n}\right)$ and $\Delta t=t_{n+1}-t_{n}$. The term $\mathrm{D}(\cdot)$, different from the standard continuous derivative denoted by $D(\cdot)$, is the discrete derivative operator. It can be shown that discrete energy conservation is obtained if condition:

$$
\mathrm{D} \Phi\left(\mathbf{q}_{n+1}-\mathbf{q}_{n}\right)=\Phi_{n+1}-\Phi_{n}
$$

is satisfied If the system has symmetries introduced by the action of a Lie group $G$ (in our case translations and/or proper rotations in $\mathbb{R}^{3}$ ), discrete conservation of symmetries is achieved if the following conditions are satisfied:

$$
\mathbb{1 D}^{G} \Phi^{\mathrm{T}}=\mathbf{0} \quad, \quad \mathbb{r}_{n+\frac{1}{2}} \mathrm{D}^{G} \Phi^{\mathrm{T}}=\mathbf{0}
$$

\section{Discrete derivatives}

In this section we will discuss some particular expressions proposed in the literature over the years for the type of problems at hand, typically multibody systems: A) Scaled midpoint gradient [3]; B) Collocation gradient [4]; C) Corrected midpoint gradient $[1,2]$; D) Partitioned discrete derivative $[1,2]$.

\section{Conclusions}

The use of a discrete derivative concept allows the systematic design of EM schemes, and several different formulas have been proposed in the literature over the years, all of them energy conserving. After a careful analysis only one of them, the scaled midpoint gradient [3], appears to comply with the requirements to produce a true EM scheme, at least for systems with finitedimensional and linear configuration spaces. Interestingly, this formula was one of the first proposed and was reported as having some problems mainly related with the energy conservation These problems can be overcome with a splitting technique, which is automatically applied when implementing the scheme with a finite-element approach, as done in the numerical experiments. The results of these experiments suggest that the scaled midpoint gradient could be indeed an optimal choice for defining stable and accurate EM schemes for solving the dynamics of constrained systems, such as those typically arising in multibody dynamics.

\section{References}

[1] Óscar González. Design and analysis of conserving integrators for nonlinear hamiltonian systems with symmetry. PhD thesis, Stanford University Department of Mechanical Engineering, 1996.

[2] Óscar González. Time integration and discrete hamiltonian systems. Journal of Nonlinear Science, 6:449-467, 1996.

[3] Alexandre J. Chorin, Thomas J.R. Hughes, Marjorie F. McCracken, and Jerrold E. Marsden. Product formulas and numerical algorithms. Communications on Pure and Applied Mathematics, 31:205-256, 1978.

[4] J.C. Simó, N. Tarnow, and K.K. Wong. Exact energy-momentum conserving algorithms and symplectic schemes for non-linear dynamics. Computer methods in Applied Mechanics and Engineering, 100:63-116, 1992.

[5] J.C. García Orden Energy and Symmetry-preserving formulation of nonlinear constraints and potential forces in multibody dynamics Nonlinear dynamics, 95(1):823-837, 2019.

[6] J.E. Marsden and T.S. Ratiu Introduction to mechanics and symmetry Springer-Verlag, 1994 